Document downloaded from:

http://hdl.handle.net/10251/137019

This paper must be cited as:

Ferri, JM.; Motoc, DL.; Ferrándiz Bou, S.; Balart, R. (2019). Thermal expansivity and degradation properties of PLA/HA and PLA/

bTCP in vitro conditioned composites. Journal of Thermal Analysis and Calorimetry (Online). 138(4):2691-2702. https://doi.org/10.1007/s10973-019-08799-0

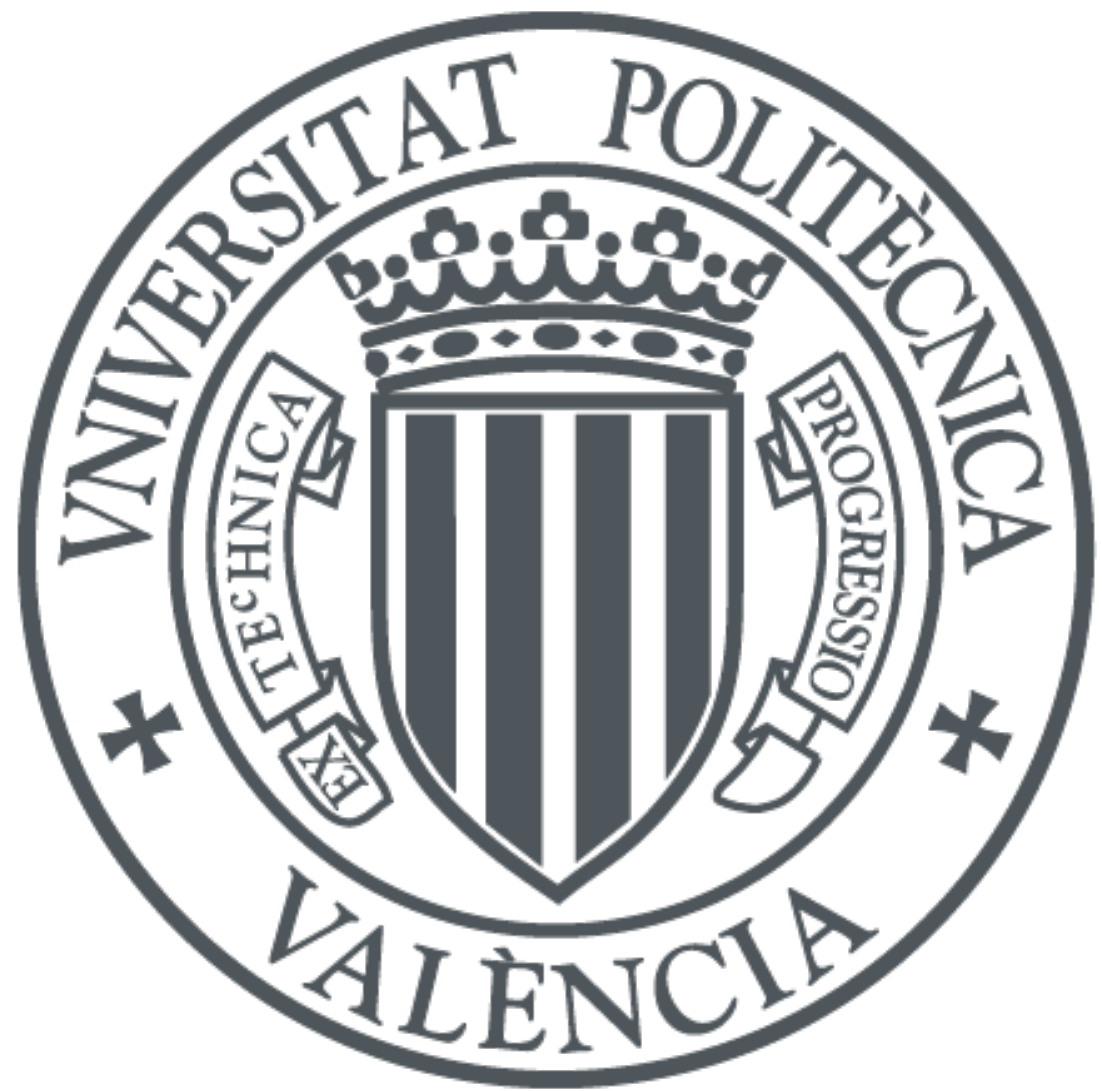

The final publication is available at

https://doi.org/10.1007/s10973-019-08799-0

Copyright Springer

Additional Information 


\title{
Thermal expansivity and degradation properties of PLA/HA and PLA/ $\beta$ TCP in vitro conditioned composites
}

\author{
J. M. Ferri ${ }^{1}$, D. Luca Motoc ${ }^{2}$, S. Ferrandiz Bou ${ }^{1}$, R. Balart ${ }^{1}$ \\ ${ }^{1}$ Department of Mechanical and Materials Engineering, Universitat Politècnica de València BE CAREFULLY with \\ AFFILIATION- Our university don't recognize the article if the affiliation isn't write like that, Plaza Ferrandiz $y$ \\ Carbonell, s/n, Alcoy (Alicante), Spain \\ ${ }^{2}$ Department of Automotive and Transport Engineering, Transilvania University of Brasov, 50017 Eroilor Av., \\ Romania
}

D. Luca Motoc (corresponding author)

Phone: (+) 40742585238

Email: danaluca@unitbv.ro ordanalucamotoc@gmail.com

\section{CHECK ENGLISH in all the manuscript}

Abstract: The objective of this study was to investigate the thermal expansivities and degradation properties for several in vitro conditioned biodegradable poly (lactic acid)/hydroxyapatite (PLA/HA) and poly (lactic acid) $/ \beta$-tricalcium phosphate (PLA/ $\beta$ TCP) composites with different wt.\% of the particle reinforcements (i.e. 10, 20 and 30). The samples were prepared by extrusion followed by injection moulding and incubated in a customized simulated body fluid at $37^{\circ} \mathrm{C}$ over $60,90,120,150$ and 180 days, respectively. Thermal expansion and degradation properties of in vitro conditioned samples, along with dynamic mechanical properties of unconditioned ones were systematically investigated through coefficients of linear thermal expansion (CLTE) and thermal strain changes, decomposition temperatures, weight changes and percent residues. The results indicated that PLA/ $\beta$ TCP composites performed better than PLA/HA composites, irrespective of their filler wt.\%, revealing high values of glass transition temperatures, around a mean value of $65^{\circ} \mathrm{C}$, both on dynamic-mechanical analysis (DMA) and dilatation (DIL) measurements but lower values on their degradation temperatures, such as $360{ }^{\circ} \mathrm{C}$. The results suggest the feasibility of tailoring high loaded osteoconductive fillers reinforced PLA composites for various medical and engineering applications.

Keywords: poly(lactic acid), hydroxyapatite, $\beta$-tricalcium phosphate, expansion, degradation 


\section{Introduction}

Poly(lactic acids) (PLAs) are the most deployed biodegradable polymers in healing products, surgical implant devices, orthopedic devices, bioresorbable scaffolds for tissue engineering, packaging, textile and environmental applications because of their versatility, good mechanical, thermal and optical properties as well as the easy of processability on conventional laboratory equipment (1-3). The interest in PLA is confirmed by extensive research papers and reviews in relation with its fabrication, processing and deployment for other combinations (4-7). Taking advantage of the individual properties of PLA and accounting that the addition of fillers may enhance the effective properties of the polymeric compounds, various combinations with micro-fillers (e.g. talc, hydroxyapatite, inorganic carbonates, kaolin, etc.) or nanofillers (e.g. carbon nanotubes, halloysite, silica, etc.) were produced.

Hydroxyapatite (HA) is of high interest being a popular biomaterial which finds use as a bone or teeth replacement and repair, hard tissue repair. Balancing between advantages such as biocompatibility, osteointegration and osteoconductive properties and disadvantages including difficulties in processing owing to fragility and brittleness, PLA/HA composites were produced with synergistic effects on their material properties (8-11). One of the main challenge when producing PLA/HA composites was the poor adhesion between the constitutive due to hydrophilicity of HA reinforcements in combination to the hydrophobic polymer matrix. The issue was overcome by modification on HA surface with silane and silane derivatives or phosphate-based solutions to enhance the dispersion within the PLA matrix and hence the interfacial adhesion $(12,13)$.

Another variation that captured researchers' attention is the $\beta$-tricalcium phosphate $(\beta \mathrm{TCP})$ that in combination with PLA was widely deployed in tissue engineering for regeneration due to their enhanced biocompatibility and biodegradability $(3,14,15)$. Despite its brittleness that confines clinical application to no-load-bearing repair and substitution, good mechanical, dynamic-mechanical and thermal properties were reported especially in highly loaded (amounts up to 30 or $40 \mathrm{wt}$ \%) composites (16) making this filler an ideal material implant. Ferri et. al. reported values in the range of 1.7 to $2.5 \mathrm{GPa}$ for the tensile modulus, 4.95 to $5 \mathrm{GPa}$ for the elastic modulus in flexure and $2.3 \mathrm{GPa}$ for the storage modulus in shear for the $30 \mathrm{wt} . \%$ fillers (17).

Addressing the osteoconductive and degradation behaviour of PLA and its composites for medical applications, in vitro simulations are demanded by immersing them into a simulated body fluid. These simulations can be done either in a static or dynamic fluid and further asses the performances of the specimens at various degradation grades $(15,18$ 20 ). Kang et al. approached the in vitro degradation under flowing conditions as well as dynamic loadings of PLA/ $\beta$ TCP composites within 4 and 6 weeks and measured the changes in mass, porosity, molecular weight and compressive strength (14). Further, Masanori et al. shown that extension of immersion time length lowers the mechanical properties due to dissolution of $\beta$ TCP from the surface (20).

In relation with the production of PLA composites, since Nature Works LLC is the main leader in the market for PLA technology and production, their biopolymers under trademark Ingeo ${ }^{\mathrm{TM}}$ are suitable for extrusion, injection moulding, solvent casting, pultrusion or hot pressing $(21,22)$. Among previously, the twin-screw method is highly preferred in PLA based composites manufacturing since enable performance increase on material properties (23). Additionally, fibre high demands for additive technologies (i.e. 3D printing) enabled high loaded PLA/HA composite filaments production (24). For example, Corcione et. al. reported one-step solvent free process for fabrication of PLA/HA with wt. $\%$ of $5,15,30$ and 50 , respectively with glass transition temperatures $\left(T_{g}\right)$ decreasing from $56.45^{\circ} \mathrm{C}$ to $52.59^{\circ} \mathrm{C}$ as the filler content increases (25). Next, Siqueira et. al. investigated the morphology and thermal properties of low loaded PLA/BTCP (1, 5 and 8 wt.\%) fibres obtained by electrospinning (26). Their results show enhanced degree of crystallinity in high filler content, small differences in the $T_{g}$ and good thermal stability from thermogravimetric measurements. Fused deposition method (FDM) is another technique deployed for PLA composites production. In a paper of Drummer et al. was investigated the processing conditions on the mechanical and thermal properties of PLA/ßTCP composites fabricated based on a FDM technique (27). Their findings point to several processing parameters that influences the mechanical properties and crystallinity of the material, such is the nozzle temperature set at $225^{\circ} \mathrm{C}$.

The aim of this contribution is to study the in vitro degradation of neat PLA, PLA/HA and PLA/ $\beta T C P$ composites manufactured by extrusion followed by an injection moulding process and to assess their dynamic-mechanical, thermal expansion and thermal stability properties accounting both different wt.\% filler content and degradation time. This systematically approach aims to bring future insights in the behaviour of in vitro degradation of neat PLA and high loaded PLA/HA and PLA/ßTCP composites to help tailoring novel bio-absorbable materials for specific application and various recipients. 


\section{Experimental research}

\subsection{Materials selection}

The matrix deployed for herein research is a NatureWorks LLC (Minnetonka, USA) poly(lactic) (PLA) biopolymer, Ingeo $^{\text {TM }} 6201 \mathrm{D}$ delivered as pellets. Both injection moulding and extrusion processes can be used owing to the intrinsic PLA material properties, such as: melt flow index $15-30 \mathrm{~g} / 10 \mathrm{~min}\left(210{ }^{\circ} \mathrm{C}\right)$, density $1.24 \mathrm{~g} \mathrm{~cm}^{-3}$ and melting temperature of $150-170{ }^{\circ} \mathrm{C}$. Further, the glass transition temperature varies between $55-60{ }^{\circ} \mathrm{C}$.

The hydroxyapatite constitutive $(\mathrm{HA})-\mathrm{Ca}_{5}(\mathrm{OH})\left(\mathrm{PO}_{4}\right)_{3}$ (Sigma-Aldrich, DE), supplied as nanoparticles with mean size $\leq 200 \mathrm{~nm}$, containing $35-40 \% \mathrm{Ca}(\mathrm{Ca} / \mathrm{P}$ ratio is 1.67$)$ and the $\beta$-tricalcium phosphate $-\mathrm{Ca}_{3}\left(\mathrm{PO}_{4}\right)_{2}\left(\mathrm{Sigma}_{\mathrm{A}} \mathrm{Aldrich}\right.$, $\mathrm{DE})$, supplied as powder with average particle size of $4 \mu \mathrm{m} \pm 2 \mu \mathrm{m}$ are acting as reinforcements for the PLA polymer matrix.

\subsection{PLA and PLA composites preparation and conditioning}

The unfilled PLA and PLA based composites were produced with different wt.\% as reported in the previous contributions $(17,28)$ and further labelled as PLA, PLA-Xx\% HA and PLA-Xx\% $\beta$ TCP to differentiate among neat, HA or $\beta$ TCP reinforced composites of different weight fraction used (i.e. 10, 20 or 30). The PLA pellets were dried prior manufacturing at $80{ }^{\circ} \mathrm{C}$ for 24 hours to remove residual moisture and reduce hydrolysis effects during their processing. For PLA/HA and PLA/ $\beta$ TCP composites with previous wt.\% contents were melt blended into a L/D relation of 24 twin-screw co-rotating extruder at $60 \mathrm{r} / \mathrm{min}$. The temperature profile was $170^{\circ} \mathrm{C}, 175^{\circ} \mathrm{C}, 177.5^{\circ} \mathrm{C}$ and $180{ }^{\circ} \mathrm{C}$ from the hopper to the die. After cooling were further pelletized and processed by injection moulding on a Meteor 270/75 machine (Mateu and Sole, ES) at $180{ }^{\circ} \mathrm{C}$. The samples were injected at a pressure of 1400 bar. The cycle time was 17 seconds and the mold temperature was $50^{\circ} \mathrm{C}$. Samples were delivered under plain rectangular shapes (standard bending specimens) with the following dimensions: $80 \times 10 \times 4 \mathrm{~mm}^{3}$, covering thus different potential testing requirements. In vitro studies were performed by immersion the sample in a in situ prepared phosphate buffered solution (SBF) (29) accounted to simulate fluid body conditions, at a constant temperature of $37{ }^{\circ} \mathrm{C}$. Fresh SBF was added into the chamber at every removing time length $(30,60,90,120.150$ and 180 days, respectively), keeping the immersion volume constant during the experiment.

\subsection{Composite samples characterization}

\section{Dynamic mechanical analysis (DMA)}

Dynamic mechanical measurements were performed by aid of a controlled stress rheometer AR-G2 (TA Instruments, New Castle, USA) at a frequency of $1 \mathrm{~Hz}$, in torsion mode, within $25^{\circ} \mathrm{C}-110^{\circ} \mathrm{C}$ temperature range, using a ${ }^{\circ} \mathrm{C}$ min ${ }^{-}$

${ }^{1}$ heating rate in the dynamic step. The experimental runs comply the ASTM D5279-13 standard procedure. Plano parallel sample dimensions were set as $40 \times 10 \times 4 \mathrm{~mm}^{3}$ and were cut from the standard bending specimens in a diamond disc cutting machine. Measurement set up and data acquisition were handled in the Rheology Advantage Instrument Control AR environment enabling information gathering for the storage modulus in shear $\left(G^{\prime}\right)$ and damping curves $(\tan \delta)$.

\section{Dilatometry (DIL)}

Specimens' expansivity was monitored by aid of a push rod dilatometer DIL 402 PC (Netzsch GmbH, D), in controlled atmosphere, within $25^{\circ} \mathrm{C}-95^{\circ} \mathrm{C}$ temperature range deploying $2.5 \mathrm{~K} \mathrm{~min}^{-1}$ heating rate in the dynamic step, followed by a $15 \mathrm{~min}$ isothermal. The experimental runs comply the ASTM E228:2011 standard procedures. Plano parallel sample dimensions were set as $25 \mathrm{~mm}$ x $5 \mathrm{~mm}$ in length and width, respectively, and were cut from the standard bending specimens in a diamond disc cutting machine. Accompanying software Proteus ${ }^{\circledR}$ enabled outcomes retrieval, such as the coefficient of linear thermal expansion (CLTE or $\alpha)$, technical alpha $\left(\alpha_{T}\right)$ inflexion points and their corresponding glass transition temperatures $\left(T_{g}\right)$, namely.

\section{Thermogravimetric analysis (TGA)}

Thermogravimetric analysis on specimens was performed by aid of a STA 449 F3 Jupiter® (Netzsch GmbH, D) at a heating rate of $10{ }^{\circ} \mathrm{C} \mathrm{min}-1$, in controlled $\mathrm{N}_{2}$ atmosphere at a $20 \mathrm{~mL} \mathrm{~min}{ }^{-1}$ flow rate. The measurements comply the ISO 11358-1:2014 procedures. Dynamic mode was deployed in the heating step within the selected $25^{\circ} \mathrm{C}-650{ }^{\circ} \mathrm{C}$ 
temperature range. Alumina crucible was used for each individual specimen excerpt. The weight loss was recorded in response to temperature increases.

\section{Scanning electron microscopy (SEM)}

Specimens morphology was investigated by aid of a field emission scanning microscope FESEM Zeiss Ultra55 (Oxford Instruments, UK) at an acceleration voltage of $2 \mathrm{kV}$. The samples were subjected to a cryofracture process by aid of liquid nitrogen. To enhance image details on the particle dispersion the samples were sputtered with platinum in a sputter coater EMITECH SC7620 (Quorum Technologies Ltd., UK). Along with the previous device, a scanning microscope (SEM) module Phenom (FEI Company, Eindoven, Netherlands) was deployed to characterize the samples surface morphology from a different perspective. In this case, the surfaces were sputtered by an Au-Pd alloy.

\section{Results and discussion}

\section{Morphological characterization of PLA/HA and PLA/BTCP composites}

Morphologies of PLA/HA and PLA/ $\beta$ TCP composites are shown in the excerpts in Fig. 1 and Fig. 2. As it can be seen from the FESEM images on the PLA/HA composites (see Fig. 1), along with increase on the wt.\% content, the surfaces are becoming rougher and more porous and the fracture process can be characterized as fragile in its essence. Additionally, HA fillers have good dispersion within the PLA matrix with enhanced filler/matrix interface at lower wt.\% contents, as further seen in Fig. 2(a)-(c). On the other hand, the FESEM images of PLA/ $\beta$ TCP (see Fig.2(d)-(c)) reveal less particle dispersion within the PLA matrix compared with their HA counterparts. The particles of $\beta$ TCP are more hydrophilic than the HA particles. As a result, aggregates of $\beta \mathrm{TCP}$ particles are generated that worsen the mechanical properties of their compounds, which behave as crack initiators (REFERENCES Ferri et al. works (PLAHA and PLA-BTCP).

\section{Dynamic mechanical properties of PLA/HA and PLA/BTCP composites}

Dynamic mechanical properties of neat PLA and PLA/HA or PLA/ßTCP composites were retrieved within selected temperature range and oscillating frequency of $1 \mathrm{~Hz}$ to provide insights in the interfacial adhesion between HA or $\beta T C P$ reinforcements and the PLA matrix in addition to SEM images.

Figure 3 shows the variation of storage modulus in shear $\left(G^{\prime}\right)$ of PLA, PLA/ $\beta$ TCP and PLA/HA composites within temperature range. As can be seen, the $G$ ' of all samples preserve approximatively a constant evolution with temperature increase below their glass transition temperature $T_{g}$. Additionally, PLA/HA and PLA/ $\beta T C P$ composites exhibit higher values of $G^{\prime}$ compared with neat PLA and ascending with increase of HA and $\beta T C P$ weight fraction thus, samples becoming stiffer. Further, the slope of the glass transition $T_{g}$ vary for PLA/HA composites, the abrupt decrease within a relatively narrow temperature range showing both an enhanced interfacial adhesion between the PLA matrix and HA reinforcements and the ability of former to withstand frequency depended mechanical loadings. However, no variation is observed in the Tg of the PLA/BTCP composites due to the non-matrix-particle interaction. In the rubbery plateau, samples' behavior follows an increased tendency. These variations can be ascribed to the cold crystallization transition and occurring at temperatures above $95^{\circ} \mathrm{C}$. The higher the content of $\beta \mathrm{TCP}$, the crystallization process occurs at a lower temperature. This indicates that the particles of $\beta \mathrm{TCP}$ act as a nucleating agent in said process. In line with above, interface adhesion between PLA matrix and HA or $\beta$ TCP fillers of different wt.\% can be further sized by exploring their corresponding damping curves $(\tan \delta)$ as plotted in Fig. 4. Generally, lower $\tan \delta$ values points toward good adhesion at matrix/filler interface due to reduction in the chain polymer mobility whereas higher values suggest weak adhesion (29). These curves are further deployed to recover glass transition temperatures $T_{g}$ of the samples under discussion. The values are, as following: PLA $-69.44{ }^{\circ} \mathrm{C}$; PLA/HA composites $-55.89{ }^{\circ} \mathrm{C}, 56.08{ }^{\circ} \mathrm{C}$ and $54.54{ }^{\circ} \mathrm{C}$; PLA/ $\beta$ TCP composites $-65.92{ }^{\circ} \mathrm{C}, 65.72{ }^{\circ} \mathrm{C}$ and $64.50^{\circ} \mathrm{C}$, respectively, in wt. $\%$ increasing order. On one side, the decrease in $\mathrm{Tg}$ values is due to a variation in the degree of crystallinity. The higher the content of HA the crystallinity decreases. Ferri et al. demonstrate by DSC that degree of crystallinity is lower as the HA content increase. On the other hand, the HA particles are hydrophilic and due to this, hydrolysis occurs in the manufacturing process due to the presence of moisture remaining in the HA particles. As a result, lactic acid oligomers are generated that plasticize the PLA matrix, making it easier for the polymer chains to move. As the HA load increases, the presence of moisture increases and larger chain cleavages occur, 
allowing greater mobility of polymer chains and $\mathrm{Tg}$ values at lower temperatures (Ferri et al. referencePLA-HA).

The slight shift in the $T_{g}$ values for each materials class can be assigned to wt.\% filler content. The height of $\tan \delta$ is associated with the mobility of amorphous region in the polymer matrix. As presented, PLA/BTCP composites exhibits sharp and high $\tan \delta$ peaks indicating that they have good structural damping properties and consequently improved capacity to absorb mechanical energy compared to neat PLA. Comparatively, PLA/HA composites not only exhibit a reduction in the sharpness and height of their $\tan \delta$ peaks but accentuated left shift of $T_{g}$ values. This indicates that the HA fillers are increasingly avoiding molecular mobility and decrease the crystallinity of the composites as the $\tan \delta$ peak additionally point out to the degree of crystallization. REMOVE THIS YELLOW SENTENCE

\section{Thermal expansion of PLA/HA and PLA/BTCP composites}

Dilatometry measurements aimed to bring supplementary information on the PLA and PLA composites behavior with temperature from thermal expansivity perspective. Thus, along with the physical alpha $\mathrm{K}^{-1}$ (CLTE - Coefficient of Linear Thermal Expansion) and thermal strain fields developed within in vitro conditioned PLA and PLA composites under discussion, technical alpha $\left(\alpha_{T}\right) \mathrm{K}^{-1}$ was additionally provided since represents a unique value within an imposed temperature values $\left(25^{\circ} \mathrm{C}-95^{\circ} \mathrm{C}\right)$ to be used as further reference.

Further insights in this thermal property was considered by collecting the CLTE values at $37{ }^{\circ} \mathrm{C}$ that corresponds to an average temperature of human body following one of the major application domain of these PLA based composites, namely bone fixtures. Table 1 lists these values for all specimens covering selected wt. $\%$ of HA and $\beta$ TCP fillers over the in vitro conditioning days. A closer look into the values, generally, reveals a decrease tendency with the increase wt.\% of the inorganic filler, both HA and $\beta$ TCP fillers for identical time conditioning range as well as, individually, with degradation time increase.

Inorganic fillers significantly reduce thermal expansion values as their wt.\% increases, statement that can be easily prove if considering the simple rule of mixture during that the polymer matrix content reduces (30). Another influencing factor can be regarded to the cooling time during the specimen preparation especially in semi-crystalline polymers whose crystallization process requires time. The latter was not investigated herein and thus was hypothetically accounted $(25,31)$.

The influence of in vitro conditioning upon expansivities of PLA and PLA based composite specimens was further investigated and it can be sized in the curves of Fig. 5 that represents excerpts from the data base. The CLTE in all specimens exhibit the same tendency over temperature with minor discrepancies due to the different wt.\% of fillers. Additionally, there are differences among HA and $\beta T C P$ reinforced PLA composites, the latter revealing the lowest variation over the temperature range. One reason for this behavior can be regarded to the random distribution of the HA and $\beta$ TCP fillers within the PLA matrix and furthermore to the increases in the agglomeration degree as the wt.\% increases. Further, intakes during the immersion within simulated fluid body pose supplementary influence since it causes both mass changes and changes in porosity (14). These can be sized better in the changes due to immersion times up to 90 days ( 3 months) followed small variations caused by further exposure to the simulated body fluid for 150 and 180 days, respectively. This is consistent with the degradation rates of the scaffolds that should match the formation of a new bone that is being shifted with one month compared with the reported time intervals, due to the high HA and $\beta$ TCP loading within the PLA matrix (15). Further, the linear expansion is higher above $45^{\circ} \mathrm{C}$ in neat PLA and PLA composites herein (above $T_{g}$ as follows) as polymer chain mobility changes due to a crystallization process.

With respect to the thermal strain developed within the PLA, these revealed identical trends in all specimens irrespective of the inorganic fillers and wt.\% deployed, as it can be seen in the excerpt plots from Fig. 6. A closer look reveals the influence of the immersion time especially upon the PLA specimen individually that is more pronounced in the 60 days conditioned sample, while the previous apply for debates on the measures.

Physical alpha curves enable glass transition temperature $T_{g}$ retrieval, as provided in Table 2 for all specimens and immersion time lengths considered. A general decreasing tendency of $T_{g}$ values in all PLA and PLA composites can be sized over the immersion time range, more pronounced in $30 \mathrm{wt} \% \mathrm{HA}$ reinforced PLA samples, from $53.9^{\circ} \mathrm{C}$ to $45.3{ }^{\circ} \mathrm{C}$ and $45.7{ }^{\circ} \mathrm{C}$, respectively. These are consistent with the previous findings from differential scanning calorimetry (DSC) measurements on unconditioned PLA/HA composites (28). On the other hand, for the PLA/ $/$ TCP in vitro conditioned samples minor changes are encountered along with their wt.\% increase as well as with immersion time. This can be related to the good particle distribution within the PLA matrix. The values fluctuate around $59{ }^{\circ} \mathrm{C}$ with two exceptions. One, holds in the highest immersion time (180 days) where the $T_{g}$ values are decreasing around $56{ }^{\circ} \mathrm{C}$ in all samples and the other in the $30 \mathrm{wt} . \%$ RTCP reinforced PLA matrix. Further, as debated in the cited 
references of the previous works of the herein research group, this can be related to the partial hydrolysis of PLA polymer chain due to the highly loaded and hydrophilic HA and $\beta T C P$ fillers that not only hydrolyze the ester groups of PLA matrix but promote a plasticization process with the formed lactic acid oligomers.

Another value of interest is technical alpha $\left(\alpha_{T}\right)$ that was computed over the temperature range against a reference temperature $\left(25^{\circ} \mathrm{C}\right.$ in this case) to enable both a quick data analysis and reference values to other studies, data base or computer simulations. Therefore, Fig. 7 shows the variation of this property for unfilled PLA, PLA/HA and PLA/BTCP composites over the immersion time. Generally, similar lowering effects hold with the addition of HA and $\beta T C P$ fillers within the PLA matrix, as previously identified. Supplementary, the preceding debates are exposed as abrupt variations over time in the PLA/HA samples and small changes as those encountered in the PLA/BTCP composites. Again, measurements on samples immersed within 90 days reveal differences that can be regarded to the maximum simulated fluid body intakes and degradation, further considered as initial points of the saturation process.

\section{Thermal degradation of PLA/HA and PLA/BTCP composites}

The thermogravimetric (TG) and derivative thermogravimetric (DTG) curves of PLA/ $\beta$ TCP excerpts corresponding to $10 \%$ and $30 \%$ filler loadings, respectively, immersed in simulated body fluid are shown in Fig. 8 and Fig. 9. All composite samples exhibit one main decomposition step under pyrolytic conditions, with onset temperatures within $300{ }^{\circ} \mathrm{C}-333{ }^{\circ} \mathrm{C}$ range, while differences are due to different filler \%wt. content.

Insights in the degradation process can be accounted from the TG characteristic temperatures of unconditioned PLA, PLA/HA and PLA/ $\beta$ TCP composites at a heating rate of $10 \mathrm{~K} \mathrm{~min}^{-1}$ as provided in Table 3 and further from the decomposition temperatures $\left(T_{d}\right)$ corresponding to $25 \%, 50 \%$ and $75 \%$ specimen mass loss as listed in Table 4 for the degraded samples. Since it is beyond of the purpose of this study to debate on degradation temperature and methods used to be identified (either maximum peak of DTG curve or temperature at $50 \%$ weight loss of the sample) to have an indication of destabilization as suggested in literature, we will focus next on the values listed in above tables (13). Thermal stability of PLAs was reported to be poor and highly sensitive to heat due to intramolecular trans-esterification resulting in the formation of lactide oligomers, carboxylic groups, etc. $(3,32)$. In this study the improved stability of in vitro conditioned samples can be explained by the manufacturing method that resulted in water removal. Supplementary, it is noteworthy to underline that highly loading the PLA matrix with HA or $\beta$ TCP results into improvements of the thermal stability of all composite systems. HA reinforced PLA composite systems revealed an increase of their onset degradation temperature with the addition of inorganic filler, from $326{ }^{\circ} \mathrm{C}$ up to $333{ }^{\circ} \mathrm{C}$ with fillers' wt.\% increase. Comparatively, $\beta$ TCP reinforced PLA composites shows a decrease in the values from $305^{\circ} \mathrm{C}$ to $297.7^{\circ} \mathrm{C}$ of the same measure, with fillers' wt.\% increase. As for the decomposition temperatures associated with the maximum peak of DTG curves, high values are identified in PLA/HA composites compared with their counterparts, PLA/ $\beta$ TCP for all \%wt. considered, resulting in improvements on the thermal stability of the former composition.

In Table 3 were provided the percentage residue for neat PLA, PLA/HA and PLA/ $\beta$ TCP composites. These values increase with the increase of the wt.\% of the fillers, either HA or $\beta \mathrm{TCP}$, the higher the amount the higher samples' thermal stability. In highly loaded PLA composites $(20,30 \mathrm{wt} . \%)$ the residues are comparable: $19.5 \%$ for PLA-20\%HA and $22.8 \%$ for PLA-20\% $\beta$ TCP, $28.95 \%$ for PLA- $20 \% \mathrm{HA}$ and $28.7 \%$ for PLA- $20 \% \beta \mathrm{TCP}$, respectively and includes small quantities of residual ashes from PLA.

Following the contribution of Schindler et al. (33) and the known correlations between glass transition and melting temperatures (34) as well as between melting and decomposition temperatures, our experimental data were shaped as illustrated in Fig. 10. The values correspond to the unconditioned PLA, PLA/HA and PLA/BTCP samples aiming to ease data comparison accounting similar values from other contributions or to further tailor these combinations.

From Fig. 10 it can be concluded that PLA polymer has the highest glass transition and melting temperatures but relatively a low decomposition temperature. This behavior does not preclude its identification by means of a dedicated software. Additionally, these plots outline better the differences debated due to filler type, either HA or $\beta$ TCP. As it can be seen, the tendency points toward a clustering effect in all wt.\% considered. PLA/BTCP composites are characterized by high values of their glass temperatures but reduced decomposition temperatures whereas PLA/HA composites reveal high values on their decomposition temperatures.

We could eliminate Figure 10 and this paragraph. This does not provide additional information. .......? 


\section{Conclusions}

The main topic of this work is the in vitro conditioning of biodegradable composites formed by PLA matrix with different wt. $\%$ HA and $\beta \mathrm{TCP}$ constitutive to determine the relevance of immersion time and fillers loading upon the thermal expansivity and thermal stability behaviour of these composites. It was found that both composite combinations show similar trends in their CTLE and thermal strain evolution with temperature, while the increase in the filler content give rise to a decrease in the measured CTLE coefficients, inherent behaviour for these materials. More pronounced effects were registered with the $\beta$ TCP filler, irrespective of the wt.\% content, giving rise to an enhanced thermal stability. Further, immersion time increase lead to an increasing tendency of CTLE values in all composites that can be regarded to the dissolution of HA and $\beta$ TCP from the surface leaving the PLA constitutive to act as the main "contributor" to the property investigated. Certainly, manufacturing process itself and processing conditions may pose an influence on the results, but further investigations were not tackled in this study.

Concerning the thermogravimetric measurements, all in vitro conditioned PLA based composite samples follow a single step decomposition process, with differences due to the filler type and content. Among all, the PLA/HA combinations revealed enhanced onset temperatures on their decomposition curves, highest mass change and relatively lowest value on the residues comparatively with their PLA/ $\beta T C P$ counterparts. The same apply with respect to the decomposition temperatures associated with the maximum peak of DTG curves.

Dynamic mechanical analysis on unconditioned PLA and PLA based composites were added to provide insights in the glass temperatures, $T_{g}$, from the peak values in the $\tan \delta$ curves. Sharp and higher peaks were found in the PLA/ $/$ TCP composites with direct consequences on their enhanced capacity to absorb mechanical energy and thus to withstand frequency depended mechanical loadings. Further data processing under decomposition over melting and melting over glass transition temperatures facilitates improved comprehension on these PLA and PLA based composites as well as comparison with other reported data from literature.

The main conclusion that can be withdrawn from the present study is that the increase of HA or $\beta$ TCP fillers content within the PLA matrix does not limit the composites material performance, even though in vitro conditioned for an extended time range. Moreover, the double step manufacturing process, extrusion followed by injection molding, is a comfortable and easy route in developing PLA, PLA/HA and PLA/ $\beta T C P$ composites with enhanced dynamicmechanical and thermal properties. These findings help to further tailor high loaded osteoconductive fillers reinforced PLA composites for various medical and engineering applications.

\section{References}

1. Auras R, Lim, Loong T., Selke, Susan, Tsuji, Hideto Poly(lactic acid): Structures, Production, Synthesis, and Applications. New York, NY: John Wiley \& Sons; 2010.

2. Murariu M, Dubois P. PLA composites: From production to properties. Advanced Drug Delivery Reviews. 2016;107:17-46.

3. Haaparanta A-M, Haimi S, Ellä V, Hopper N, Miettinen S, Suuronen R, et al. Porous polylactide/ $\beta$-tricalcium phosphate composite scaffolds for tissue engineering applications. Journal of Tissue Engineering and Regenerative Medicine. 2010;4(5):366-73.

4. Ahmed J, Varshney SK. Polylactides-Chemistry, Properties and Green Packaging Technology: A Review. International Journal of Food Properties. 2011;14(1):37-58.

5. Garlotta D. A Literature Review of Poly(Lactic Acid). Journal of Polymers and the Environment. 2001;9(2):63-84. 6. Slomkowski S, Penczek S, Duda A. Polylactides - an overview. Polymers for Advanced Technologies. 2014;25(5):436-47.

7. Avinc O, Khoddami A. Overview of Poly(lactic acid) (PLA) Fibre. Fibre Chemistry. 2009;41(6):391-401.

8. Akindoyo JO, Beg MDH, Ghazali S, Heim HP, Feldmann M. Impact modified PLA-hydroxyapatite composites Thermo-mechanical properties. Composites Part A: Applied Science and Manufacturing. 2018;107:326-33.

9. Nazhat SN, Kellomäki M, Törmälä P, Tanner KE, Bonfield W. Dynamic mechanical characterization of biodegradable composites of hydroxyapatite and polylactides. Journal of Biomedical Materials Research. 2001;58(4):335-43.

10. Ignjatovic N, Uskokovic D. Synthesis and application of hydroxyapatite/polylactide composite biomaterial. Applied Surface Science. 2004;238(1):314-9.

11. Li J, Zheng W, Li L, Zheng Y, Lou X. Thermal degradation kinetics of g-HA/PLA composite. Thermochimica Acta. 2009;493(1):90-5.

12. Zhang SM, Liu J, Zhou W, Cheng L, Guo XD. Interfacial fabrication and property of hydroxyapatite/polylactide resorbable bone fixation composites. Current Applied Physics. 2005;5(5):516-8. 
13. Akindoyo JO, Beg MDH, Ghazali S, Heim HP, Feldmann M. Effects of surface modification on dispersion, mechanical, thermal and dynamic mechanical properties of injection molded PLA-hydroxyapatite composites. Composites Part A: Applied Science and Manufacturing. 2017;103:96-105.

14. Kang Y, Yao Y, Yin G, Huang Z, Liao X, Xu X, et al. A study on the in vitro degradation properties of poly(1lactic acid)/ $\beta$-tricalcuim phosphate(PLLA/ $\beta$-TCP) scaffold under dynamic loading. Medical Engineering \& Physics. 2009;31(5):589-94.

15. Huang J, Ten E, Liu G, Finzen M, Yu W, Lee JS, et al. Biocomposites of pHEMA with HA/ $\beta$-TCP (60/40) for bone tissue engineering: Swelling, hydrolytic degradation, and in vitro behavior. Polymer. 2013;54(3):1197-207.

16. Bleach NC, Nazhat SN, Tanner KE, Kellomäki M, Törmälä P. Effect of filler content on mechanical and dynamic mechanical properties of particulate biphasic calcium phosphate-polylactide composites. Biomaterials. 2002;23(7):1579-85.

17. Ferri J, Gisbert I, García-Sanoguera D, Reig M, Balart R. The effect of beta-tricalcium phosphate on mechanical and thermal performances of poly(lactic acid). 2016;50(30):4189-98.

18. Li X, Qi C, Han L, Chu C, Bai J, Guo C, et al. Influence of dynamic compressive loading on the in vitro degradation behavior of pure PLA and Mg/PLA composite. Acta Biomaterialia. 2017;64:269-78.

19. Agrawal CM, McKinney JS, Lanctot D, Athanasiou KA. Effects of fluid flow on the in vitro degradation kinetics of biodegradable scaffolds for tissue engineering. Biomaterials. 2000;21(23):2443-52.

20. Kikuchi M, Koyama Y, Takakuda K, Miyairi H, Shirahama N, Tanaka J. In vitro change in mechanical strength of $\beta$-tricalcium phosphate/copolymerized poly-L-lactide composites and their application for guided bone regeneration. Journal of Biomedical Materials Research. 2002;62(2):265-72.

21. Lim LT, Auras R, Rubino M. Processing technologies for poly(lactic acid). Progress in Polymer Science. 2008;33(8):820-52.

22. Ignjatovic N, Suljovrujic E, Budinski-Simendic J, Krakovsky I, Uskokovic D. Evaluation of hot-pressed hydroxyapatite/poly-L-lactide composite biomaterial characteristics. Journal of Biomedical Materials Research Part B: Applied Biomaterials. 2004;71B(2):284-94.

23. Martin C. Twin Screw Extrusion for Pharmaceutical Processes. In: Repka MA, Langley N, DiNunzio J, editors. Melt Extrusion: Materials, Technology and Drug Product Design. New York, NY: Springer New York; 2013. p. 4779.

24. Cox SC, Thornby JA, Gibbons GJ, Williams MA, Mallick KK. 3D printing of porous hydroxyapatite scaffolds intended for use in bone tissue engineering applications. Materials Science and Engineering: C. 2015;47:237-47.

25. Corcione C, Scalera F, Gervaso F, Montagna F, Sannino A, Maffezzoli A. One-step solvent-free process for the fabrication of high loaded PLA/HA composite filament for 3D printing. Journal of Thermal Analysis and Calorimetry. 2018:1-8.

26. Siqueira L, Passador FR, Costa MM, Lobo AO, Sousa E. Influence of the addition of $\beta$-TCP on the morphology, thermal properties and cell viability of poly (lactic acid) fibers obtained by electrospinning. Materials Science and Engineering: C. 2015;52:135-43.

27. Drummer D, Cifuentes-Cuéllar S, Rietzel D. Suitability of PLA/TCP for fused deposition modeling. Rapid Prototyping Journal. 2012;18(6):500-7.

28. Ferri J, Jordá J, Montanes N, Fenollar O, Balart R. Manufacturing and characterization of poly(lactic acid) composites with hydroxyapatite. Journal of Thermoplastic Composite Materials. 2018;31(7):865-81.

29. Menczel JD, Prime RB. Thermal Analysis of Polymers: Fundamentals and Applications: Wiley; 2014.

30. Aboudi J, Arnold SM, Bednarcyk BA. Chapter 3 - Fundamentals of the Mechanics of Multiphase Materials. In: Aboudi J, Arnold SM, Bednarcyk BA, editors. Micromechanics of Composite Materials. Oxford: ButterworthHeinemann; 2013. p. 87-145.

31. Esposito Corcione C, Gervaso F, Scalera F, Padmanabhan SK, Madaghiele M, Montagna F, et al. Highly loaded hydroxyapatite microsphere/ PLA porous scaffolds obtained by fused deposition modelling. Ceramics International. 2018.

32. Zou H, Yi C, Wang L, Liu H, Xu W. Thermal degradation of poly(lactic acid) measured by thermogravimetry coupled to Fourier transform infrared spectroscopy. Journal of Thermal Analysis and Calorimetry. 2009;97(3):929.

33. Schindler A, Doedt M, Gezgin Ş, Menzel J, Schmölzer S. Identification of polymers by means of DSC, TG, STA and computer-assisted database search. Journal of Thermal Analysis and Calorimetry. 2017;129(2):833-42.

34. Lee WA, Knight GJ. Ratio of the glass transition temperature to the melting point in polymers. British Polymer Journal. 1970;2(1):73-80.

\section{Figure caption}


Fig. 1 FESEM images at $\mathrm{x} 10,000$ on cryofractured surfaces of PLA/HA composites with different wt.\% hydroxyapatite: (a) neat PLA, (b) $10 \mathrm{wt} \% \% \mathrm{HA}$, (c) $20 \mathrm{wt} \% \% \mathrm{HA}$, and (d) $30 \mathrm{wt} . \% \mathrm{HA}$

Fig. 2 SEM images of PLA composites with different wt.\% fillers: (a) 10 wt.\% HA, (b) 20 wt.\% HA, (c) 30 wt.\% HA, (d) $10 \mathrm{wt} . \% \beta \mathrm{TCP}$, (e) $20 \mathrm{wt} . \% \beta \mathrm{TCP}$ and (f) $30 \mathrm{wt} . \% \beta \mathrm{TCP}$

Fig. 3 Storage modulus $\left(G^{\prime}\right)$ over temperature range of unconditioned PLA/ $\beta T C P$ composites CHANGE

Fig. 4 Damping curves of unconditioned neat PLA, PLA/HA and PLA/ $\beta$ TCP composites

Fig. 5 Physical alpha over temperature range of in vitro conditioned neat PLA, PLA/HA and PLA/ $\beta$ TCP composites $(60,90,150,180$ days $)$

Fig. 6 Thermal strain over temperature range of in vitro conditioned neat PLA, PLA/HA and PLA/ $\beta$ TCP composites $(60,90,150,180$ days $)$

Fig. 7 Technical alpha variation with in vitro conditioning time of PLA, PLA/HA and PLA/ $\beta$ TCP composites

Fig. 8 Temperature dependent mass change (TG) of in vitro conditioned 10 wt. \% and 30 wt.\% PLA/ $\beta$ TCP composites

Fig. 9 Rate of mass change (DTG) of in vitro conditioned 10 wt.\% and 30 wt.\% PLA/ $\beta$ TCP composites

Fig. 10 Glass transition, melting and decomposition temperatures of unconditioned neat PLA, PLA/HA and PLA/ $\beta$ TCP composites

\section{Table caption}

Table 1 - CLTE $\left(\cdot 10^{-5} \mathrm{~K}^{-1}\right)$ of PLA, PLA-HA and PLA- $\beta$ TCP in vitro conditioned samples, retrieved at temperature of $37^{\circ} \mathrm{C}$

Table 2 - Tg $\left({ }^{\circ} \mathrm{C}\right)$ values of PLA, PLA-HA and PLA- $\beta$ TCP in vitro conditioned samples from DIL measurements

Table 3 - Thermal degradation results of PLA, PLA-HA and PLA- $\beta$ TCP samples

Table 4 - Decomposition temperatures for PLA, PLA-HA and PLA- $\beta$ TCP samples for different mass loss values 